\title{
PERFORMANCE EVALUATION OF DSDV, AODV ROUTING PROTOCOLS IN VANET
}

\author{
K. Venkateswarlu ${ }^{1}$, G. Murali $^{2}$ \\ ${ }^{I}$ M. Tech, CSE, JNTUA College of Engineering (Pulivendula), Andhra Pradesh, India \\ ${ }^{2}$ Asst.Prof (HOD), CSE, JNTUA College of Engineering (Pulivendula), Andhra Pradesh, India
}

\begin{abstract}
Wireless networks between vehicles formed by VANET (Vehicular Ad hoc Networks). VANET incorporates the wireless communication and data sharing capabilities to turn into vehicles as a network topology. VANET is showing great potential in research area. Inter-vehicle communication system in VANET improves traffic safety. VANET consists of high dynamic topology, Intermittent connectivity Patterned mobility and on board sensors etc. A challenging task in vehicular ad hoc network is routing of data due to its high dynamic behavior. An efficient routing protocol by understanding the dynamic behavior of VANET topology will plays an essential role in communication of vehicles. Mobility of nodes, road topologies are some of the internal factors are presented on the performance of routing protocols. Routing protocols are of mainly categorized into: Proactive, Reactive and Hybrid routing protocols. This paper occurs with performance evaluation of AODV (Ad-Hoc on-Demand Distance Vector), DSDV (Destination-Sequenced Distance Vector) routing protocols using mobility model IDM-IM (Intelligent Driver Model with Intersection Management, this mobility model is from VanetMobisim) based on metrics such as packet delivery ratio, throughput and average end to end delay. AODV is reactive routing protocol and DSDV is proactive routing protocol. IDM_IM is the VanetMobisim tool mobility model with a simulation area. In this paper we also present how the VanetMobisim interacts with Network Simulator. VanetMobisim presents results as a text file. The simulation results are presented with graphs. VanetMobisim and Network Simulator tools are open access tools. VanetMobisim a vehicular ad hoc network tool and network simulator-2(NS2) a simulation tool are used for implementation purpose.
\end{abstract}

Keywords: VANET (Vehicular Ad hoc Networks), AODV, DSDV, VanetMobisim, NS2

\section{INTRODUCTION}

A promising research area to improve road safety by developing intelligent vehicular-inter communication is VANET. VANETS are combination of mobile nodes, vehicles equipped with On Board Unit (OBU), and Stationary nodes called Road Side Units (RSU) attached to infrastructure. The OBU and RSU will have wired/wireless communication capabilities. Like ad-hoc manner OBUs communicate with each other and also with RSUs [2]. Basically Vanet is two kinds of communication environments [3]: Vehicle to Vehicle (V2V) and Vehicle to Road (V2R) communications.

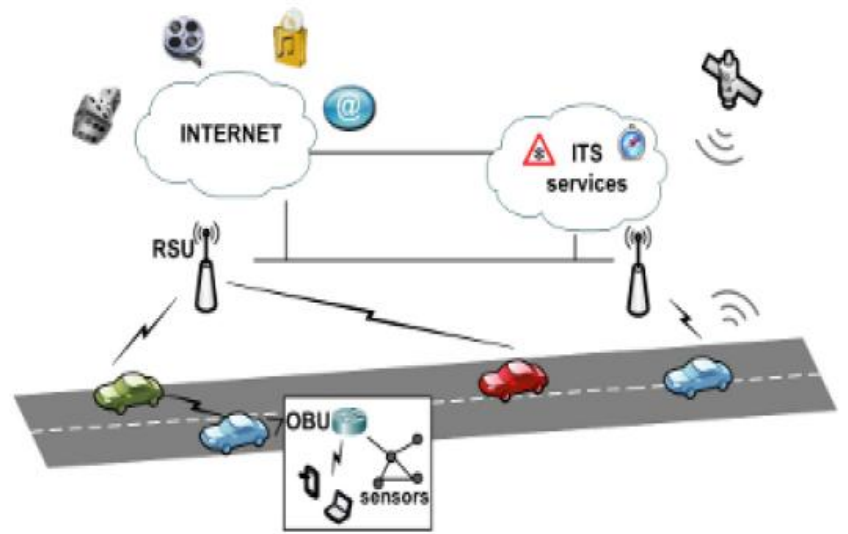

Fig -1: Vehicular ad-hoc network
In Vanet not only vehicle to vehicle communication also vehicle to infrastructure even RSUs also communicate with each other. Vanet communication allows various types of applications. These are mainly categorized as safety applications, comfort applications and Administrator applications [4].

The key issue in Vanet is routing. A specific choice of route is established using routing algorithms. In this paper we present one proactive (DSDV) routing protocol algorithm and one reactive (AODV) routing protocol algorithm.

The rest of the paper is organized as below: Distinction 2 describes characteristics of Vanet. Distinction 3 describes mobile ad hoc routing protocols categories. Distinction 4 describes simulation area and measurements of metrics and then results are presented in Distinction 5. At last in Distinction 6 we conclude the paper.

\section{VEHICULAR AD HOC NETWORKS CHARACTERISTICS}

Some common properties of Manet are also presented Vehicular ad hoc network (VANET), but there are some individually distinct characteristics in VANET. 
Some of these characteristics can be presented as below [5]: 1. Great mobility: In Vanet due to high mobility of nodes means relative speed is high so change in topology is very fast

2. Mobility patterns as restricted and predictable: Vanet networks have some restricted rules for node movements; these rules are to be theory rules of traffic flow. These restricted rules make Vanets as predictable at least on the short run.

3. No power constrains: The battery which used as an infinite power supply for communications and computation task is equipped with each vehicle.

4. Localization: With use of Global Positioning System (GPS) in Vanet it is easy to identify locations of vehicles with high accuracy.

\section{ROUTING PROTOCOLS (RPs)}

Mobile ad hoc network routing protocols are categorized into two main categories (Fig 2):

1. Proactive routing protocols: These routing protocols maintain all nodes information in the topology irrespective of the node whether they are participating or not in the communication. The information of network topology in these protocols is discovered by periodic control packets. To maintain the routing information, the information is transmitted throughout the network.

2. Reactive routing protocols: In reactive routing protocols whenever a node wants to send packets to its destination node then only node initiates a route discovery throughout the network. Source routing and hop-by-hop routing is presented in reactive routing protocols. In these reactive routing protocols route is maintained by route maintenance process. The route maintenance process is presented until destination inaccessible or until the route is no longer presented [6].

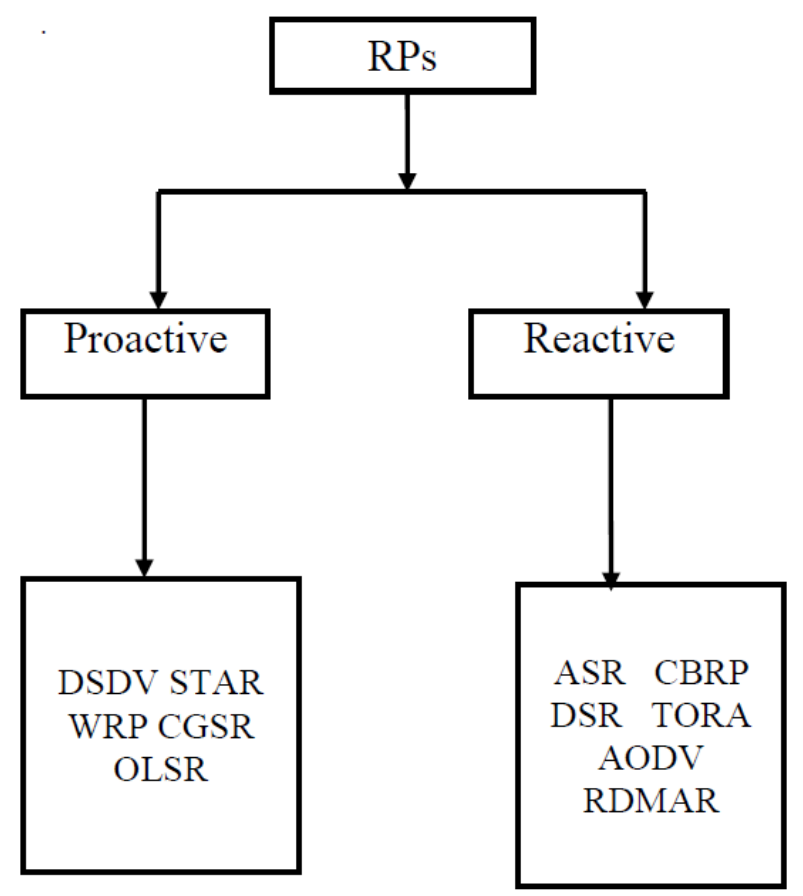

Fig.2 Routing Protocols for Mobile ad hoc networks
Here we discuss about one reactive and one proactive routing protocol such as DSDV a proactive routing protocol and AODV a reactive routing protocol:

1. DSDV: To calculate the path Destination-Sequenced Distance Vector protocol uses Bellman-Ford algorithm. DSDV is a table driven routing protocol. With entries of all nodes in the network DSDV maintains a routing table. Periodic and trigger mechanisms are maintained by DSDV to propagate the changes. A sequence number is tagged for each update from the node to avoid looping in routes. Sequence number is always even whenever normal update, if a node wants to send an expired route to its neighbor node then the sequence number of disconnected node is incremented by 1 . If the sequence number is odd to a node, then it will remove from the routing table. One node cannot change other nodes sequence number.

2. AODV: Ad hoc ON - demand Distance Vector Routing protocol is a unicast reactive routing protocol for MANETs. AODV only maintains the active path information only. Routing information is maintained by routing tables at all nodes in the active path. The next hop routing table at all nodes contains the information of destinations to which the route is presented currently. Whenever the route is not been used or not reactivated for a pre-specified expiration time then routing table entry expires [7].

Unlike DSDV routing protocol, in AODV when a node wants to send a packet to a destination then only it determines a route. As long as routes are needed by the sources until routes are maintained in AODV routing protocol.

\section{ENVIRONMENT SIMULATION}

For this study, we used a combination of two tools which are a traffic simulator VanetMobisim [8] and a network simulator Ns-2 [9]. VanetMobisim is a traffic simulator and an open source platform specific for VANETs. Network simulator is also an open source platform and is useful to generate simulation results.

VanetMobisim supports both micro-mobility level and macro-mobility level specifications in VANET. Micromobility model level represents vehicles slow, planned route etc. Macro-mobility level represents acceleration, deceleration, car following each other etc. A mobility trace is generated as output with VanetMobisim tool; this mobility trace is used by a simulation tool such as ns-2 to simulate realistic vehicle movement [10].

In this paper we check the performance of AODV, DSDV routing protocols with the mobility model IDM_IM [11] for Vanet environment.

\section{IDM_IM:}

IDM_IM (Intelligent Driver Model with Intersection Management) is one of the mobility models in VanetMobisim tool. It is generated by cars in a city centre using the space graph. In this mobility model for node's motion it uses an instance of polito.uomm.IDM_IM. In this 
model based on movements of neighboring vehicles it regulates vehicles speed. This mobility model considers only vehicles moving according to the IDM_IM. The implementation reflects restrictions of the spatial environment. In IDM_IM vehicles will slow down and stop at intersections or act according to traffic light if present.

For this paper we used 29 vehicles. With dimensions $1000 * 1000 \mathrm{~m} 2$. Node speed movements would be specified as minimum speed as $10.0 \mathrm{~m} / \mathrm{s}$ and maximum speed as 20.0 $\mathrm{m} / \mathrm{s}$. Simulation time taken as 2000.0 . In this mobility model node's trip and node's initial potion would be specified with instances as tripgenerator, initposgenerator. Initial stay duration also specified in this mobility model minimum stay as 20.0 and max stay as 100.0.

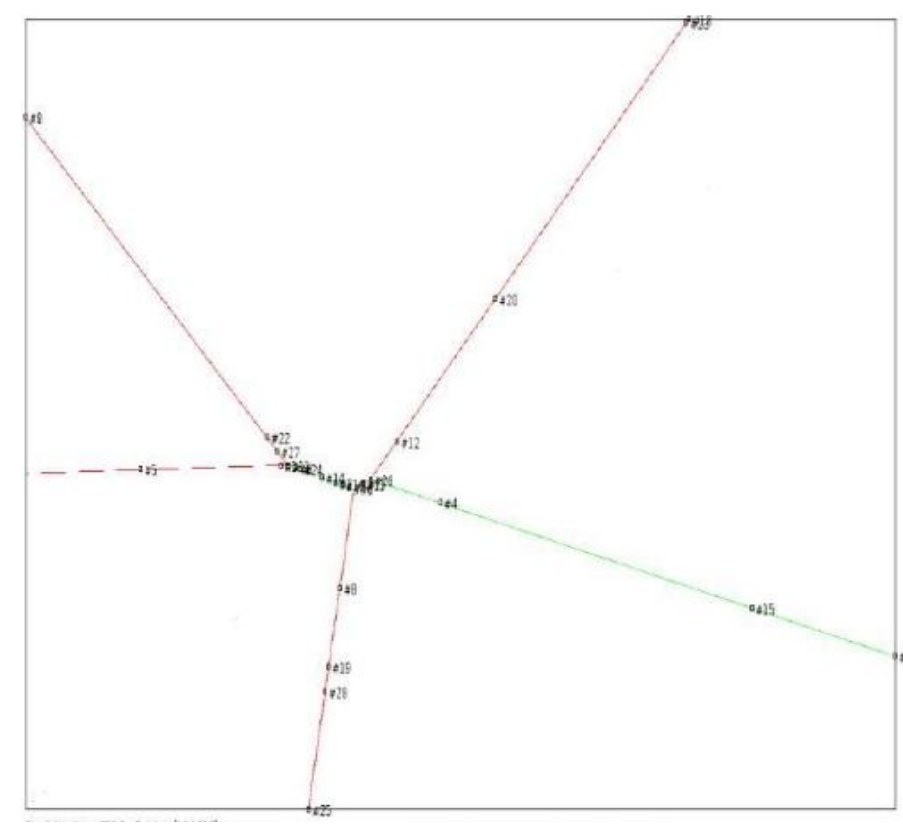

Fig.3 IDM_IM Scenario (VanetMobisim)

The IDM_IM simulation environment is presented in Figure 3. This also results a traffic trace file as a text file.

Table 1: VanetMobisim feature scenario

\begin{tabular}{|l|l|}
\hline Dimension $\left(\mathrm{m}^{2}\right)$ & $1000 * 1000$ \\
\hline Number Nodes & 29 \\
\hline Speed Nodes & Min 10.0, Max 20.0 \\
\hline Driver Model & IDM_IM.xml \\
\hline Simulation Time & 2000.0 \\
\hline
\end{tabular}

\section{Network Simulator:}

For Network Simulator we use NS-2.35 an open source tool. It is discrete event simulator. A substantial support is provided by NS-2 for routing, simulation of TCP and multicast protocols. It provides support for both wireless and wired networks. In Table. 2 simulation area specifications are presented for NS2

Table 2: NS2 Simulation Settings

\begin{tabular}{|l|l|}
\hline Simulation Time & $250 \mathrm{~s}$ \\
\hline Number of Nodes & 29 \\
\hline
\end{tabular}

\begin{tabular}{|l|l|}
\hline Traffic Agent & TCP/FTP \\
\hline MAC Protocol & IEEE $802.11 \mathrm{p}$ \\
\hline Routing Protocol & DSDV,AODV \\
\hline
\end{tabular}

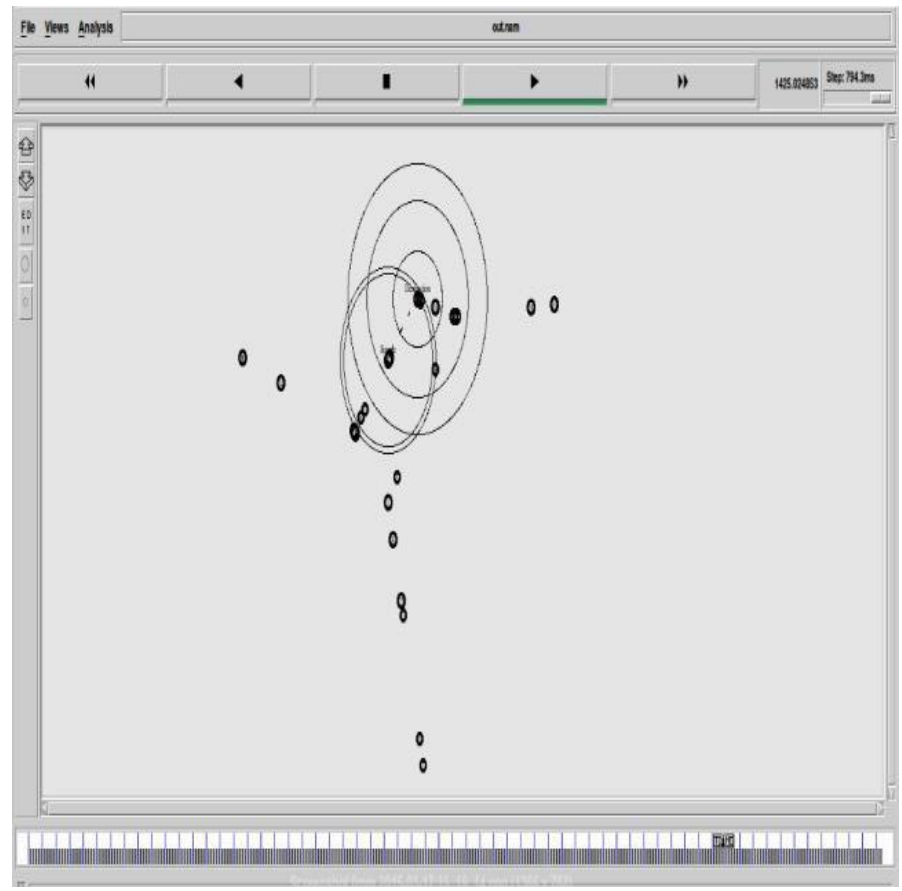

Fig.4 Simulation environment in NS2 (NAM)

Interaction between VanetMobisim and Network Simulator is specified as first we use IDM_IM traffic scenario as a .xml file to input for VanetMobisim. This generates a traffic trace file as a text file which contains nodes starting positions, destinations for all nodes and minimum, maximum speed of mobile nodes and also uses traffic specifications for mobile nodes stay. Later we use this traffic trace file along with TCL file, which is a network scenario as input for NS2 which generates network trace file. With this network trace file we produce our required information.

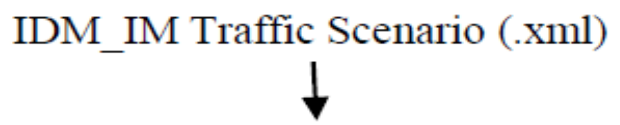

VanetMobisim

Traffic Trace File
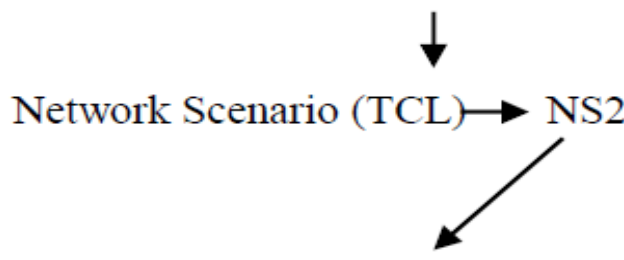

Network Trace File (.tr)

Fig.5 Simulation of Vanet based on combination of both VanetMobisim and NS2

Figure 5 shows how coupling of both VanetMobisim and NS2 is done. 4 


\section{RESULTS AND ANALYSIS:}

Here we selected two routing protocols one is proactive DSDV routing protocol and another one is reactive routing protocol, for these two we calculated metrics such as Packet Delivery Ratio, Throughput and Average end to end Delay these are performance metrics for a routing protocol.

Packet Delivery Ratio - a ratio between numbers of packets received by application layer of a node to the number of packets transmitted from another node.

Throughput - average of successful message transmission over a time interval.

Average E2E Delay - the data packets average time delay from source node to destination node is average e2e delay

In Figure 6, the packet delivery ratio of DSDV and AODV protocols are specified. The number of vehicles in this scenario was 29. The simulation time varies from 250 to 1450 with an interval of 300 . We observe that the PDR of both DSDV and AODV decrease from low simulation timings to higher simulation timings. In graph the horizontal axis represents simulation time and the vertical axis represents packet delivery ratio.

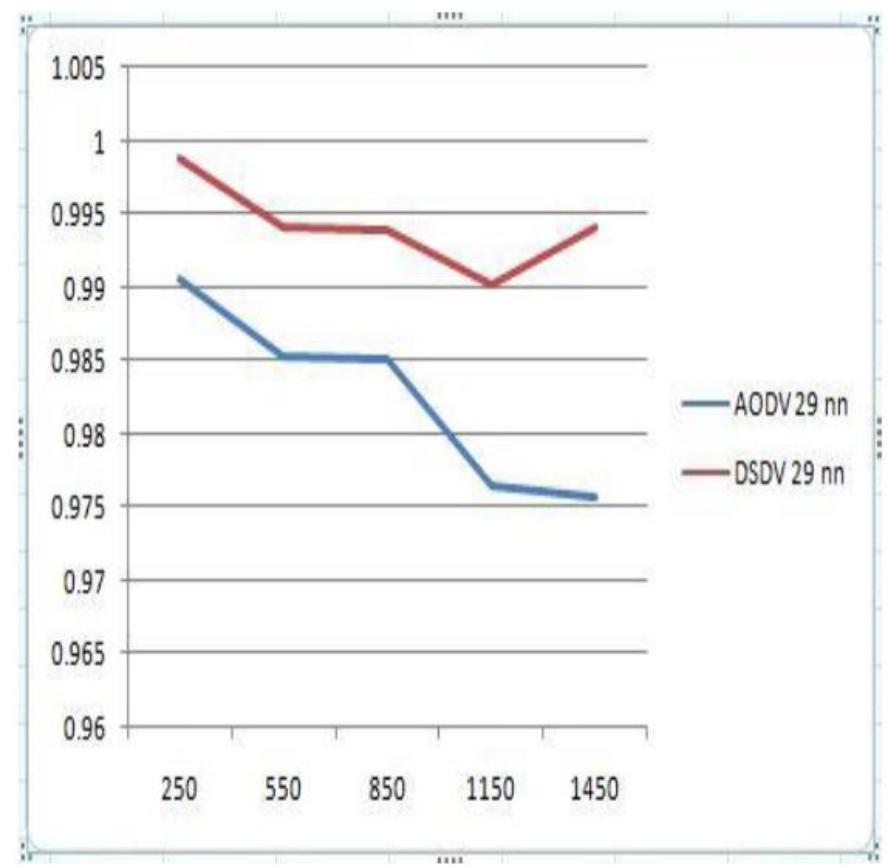

Fig 6: PDR versus Simulation time (IDM_IM scenario)

In Figure 7, the throughput of DSDV and AODV protocols are specified. The number of vehicles in this scenario was 29. The simulation time varies from 250 to 1450 with an interval of 300. In graph the horizontal axis represents simulation time and the vertical axis represents throughput in bits/sec. Here AODV exhibited the highest throughput compared to DSDV routing protocol and also some fluctuations are presented in both protocols for different simulation times.

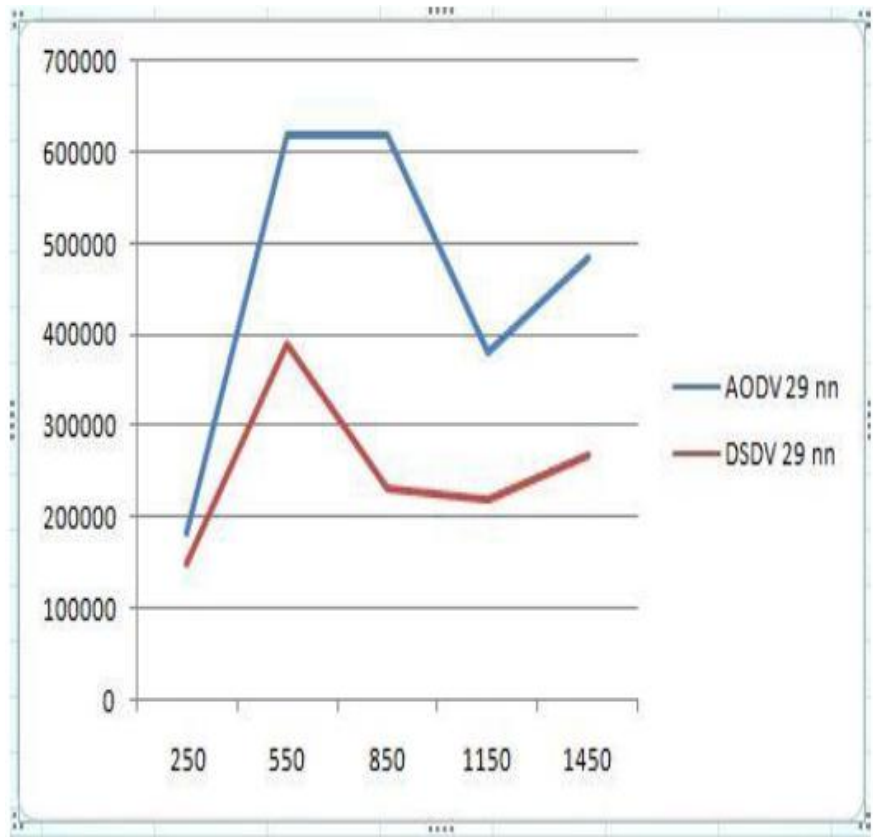

Fig 7: Throughput versus Simulation time (IDM_IM scenario)

In Figure 8, the e2e delay of DSDV and AODV protocols are specified. The number of vehicles in this scenario was 29. The simulation time varies from 250 to 1450 with an interval of 300. In graph the horizontal axis represents simulation time and the vertical axis represents e2edelay in $\mathrm{m} / \mathrm{s}$. In this observation we observed that DSDV routing protocol outperforms AODV in terms of End to End delay.

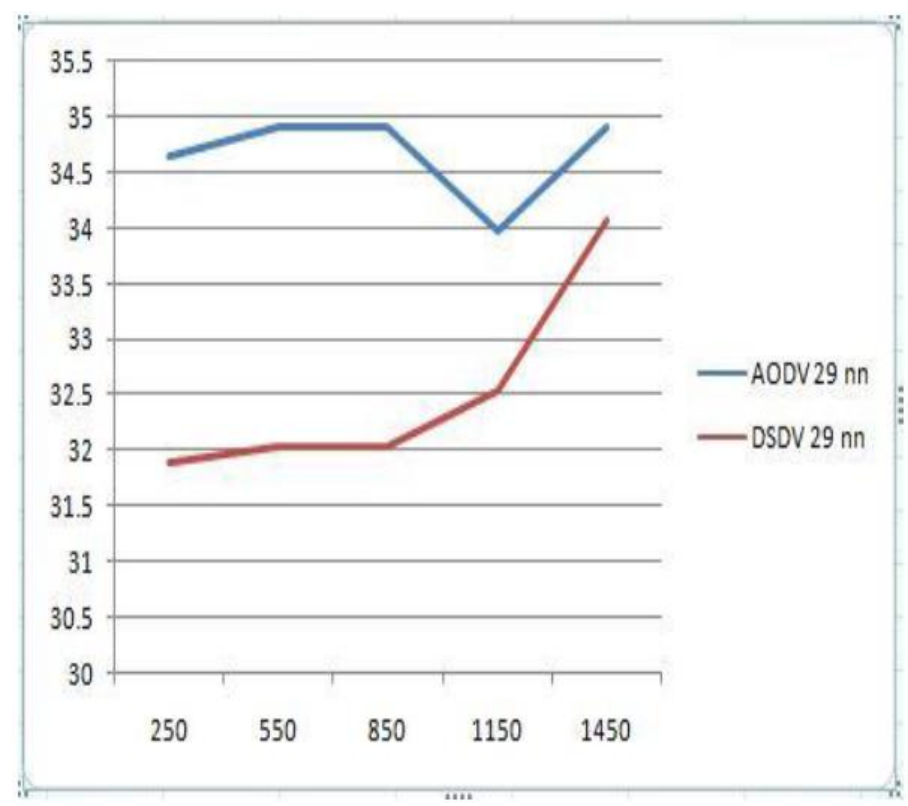

Fig 8: E2E Delay versus Simulation time (IDM_IM scenario)

From Figure 6 to Figure 8 the results are shown in graph these all results are generated by coupling both VanetMobisim and Network Simulator. 


\section{CONCLUSION}

In this work, we have presented the metric results for performance evaluation of two routing protocols such as DSDV and AODV with less number of vehicles by using mobility model IDM_IM in terms of packet delivery ratio, throughput and end to end delay. The results show that: DSDV outperforms AODV for small network as less number of vehicles. In future we consider large network with more number of vehicles.

\section{REFERENCES}

[1]. M. Guezouri and A. Bennaoui, Performance Evaluation of Routing Protocols in Vehicular Networks. I. J. Computer Network and Information Security, 2013, 10, 11-16

[2]. H. Hartenstein and K. Laberteaux, VANET - Vehicular Applications and Inter-Networking Technologies. Chapter 7, Wiley, ISBN 978-0-470-74056-9, pp. 219-272, 2010.

[3]. Yogesh Suryawanshi, Avichal Kapur ,Analysis of Routing Protocol in Vehicular Ad hoc Network. International Journal of Advanced Engineering Sciences and Technologies, Vol no. 8, issue no. 2, $281-285$.

[4]. Roger Calzada. ,Performance evaluation of realistic scenarios for vehicular Ad Hoc networks with VanetMobiSim and ns2. Proyecto final de carrera, UPC, 2011.

[5]. Zaydoun Yahya Rawashdeh and Syed Masud Mahmud. Communications in Vehicular Ad Hoc Networks, Mobile Ad-Hoc Networks: Applications, Xin Wang (Ed.), ISBN: 978-953-307-416-0, InTech, DOI: 10.5772/13399, 2011.

[6]. Anuj K., Gupta Harsh Sadawarti and Anil K. Verma, Performance analysis of AODV, DSR \& TORA Routing Protocols ,IACSIT International Journal of Engineering and Technology, Vol.2, No.2, April 2010 ISSN: 1793-8236.

[7]. Yun-Wei Lin, Yuh-Shyan Chen and Sing-Ling Lee. Routing in Vehicular Ad Hoc Networks: A Survey and Future Perspectives,Journal of Information Science and Engineering,2010.

[8]. http://vanet.eurecom.fr.

[9]. NS-2 Network simulator http://www.isi.edu/nsnam/ns.

[10]. CANU Research Group (Stuttgart University) http://canu.informatik.uni-stuttgart.de/mobisim/.

[11]. IDM-IM (Intelligent Driver Model with Intersection Management) VanetMobisim manual. 